Groups Geom. Dyn. 5 (2011), 107-119

DOI $10.4171 / \mathrm{GGD} / 117$
Groups, Geometry, and Dynamics

(C) European Mathematical Society

\title{
On Beauville surfaces
}

Yolanda Fuertes, Gabino González-Diez and Andrei Jaikin-Zapirain

\begin{abstract}
We prove that if a finite group $G$ acts freely on a product of two curves $C_{1} \times C_{2}$ so that the quotient $S=C_{1} \times C_{2} / G$ is a Beauville surface then $C_{1}$ and $C_{2}$ are both non hyperelliptic curves of genus $\geq 6$; the lowest bound being achieved when $C_{1}=C_{2}$ is the Fermat curve of genus 6 and $G=(\mathbb{Z} / 5 \mathbb{Z})^{2}$. We also determine the possible values of the genera of $C_{1}$ and $C_{2}$ when $G$ equals $S_{5}, \mathrm{PSL}_{2}\left(\mathbb{F}_{7}\right)$ or any abelian group. Finally, we produce examples of Beauville surfaces in which $G$ is a $p$-group with $p=2,3$.
\end{abstract}

Mathematics Subject Classification (2010). 14J29, 20D06, 30F10.

Keywords. Beauville surfaces, Riemann surfaces, finite $p$-groups.

\section{Introduction and statement of results}

A complex algebraic curve $C$ will be termed triangle curve if it admits a finite group of automorphisms $G<\operatorname{Aut}(C)$ so that $C / G \cong \mathbb{P}^{1}$ and the natural projection $C \rightarrow C / G$ ramifies over three values, say $0,1, \infty$. If the branching orders at these points are $p, q$ and $r$ we will say that $C / G$ is an orbifold of type (or signature) $(p, q, r)$ or, more simply, that $C / G$ is of type $(p, q, r)$. Triangle curves are also known as arithmetic curves or regular Belyi (Riemann) surfaces. This is because of the following theorem

Belyi's Theorem. A complex algebraic curve $C$ can be defined over a number field if and only if there is a (Belyi) function $f: C \rightarrow \mathbb{P}^{1}$ ramified over three values.

For complex surfaces $S$ an analogous criterion in which Belyi functions are replaced by Lefschetz functions is given in [6]. Among the complex surfaces defined over a number field an important class is that of Beauville surfaces defined as follows

Definition ([3]). A Beauville surface is a compact complex surface $S$ satisfying the following properties:

1) It is isogenous to a higher product, that is $S \cong C_{1} \times C_{2} / G$, where $C_{i}(i=1,2)$ are curves of genera $g_{i} \geq 2$ and $G$ is a finite group acting freely on $C_{1} \times C_{2}$ by holomorphic transformations. 
2) If $G^{o}<G$ denotes the subgroup consisting of the elements which preserve each of the factors, then $G^{o}$ acts effectively on each curve $C_{i}$ so that $C_{i} / G^{o} \cong \mathbb{P}^{1}$ and $C_{i} \rightarrow C_{i} / G^{o}$ ramifies over three points.

It is easy to see ( [5]) that an automorphism of the product of two curves as above $f: C_{1} \times C_{2} \rightarrow C_{1} \times C_{2}$ either preserves each factor or interchanges them. Clearly, if two such automorphisms $f_{1}, f_{2}$ interchange the factors, which can only occur when $C_{1} \cong C_{2}$, then $f_{1} \circ f_{2}$ will preserve them, hence $G^{o}$ has at most index 2 in $G$. A Beauville surface $C_{1} \times C_{2} / G$ is said to be of mixed or unmixed type according to whether $\left[G: G^{o}\right]=2$ or $G=G^{o}$. Accordingly the group $G$ is said to admit a mixed or unmixed Beauville structure.

It is clear that any Beauville surface of mixed type $S=C_{1} \times C_{2} / G$ automatically gives rise to a Beauville surface of unmixed type $S^{o}=C_{1} \times C_{2} / G^{o}$. This is the reason why for the purpose of this article Beauville surfaces of mixed type will play no role.

Beauville surfaces were introduced by F. Catanese in [5] generalizing the following construction of A. Beauville which appears as exercise number 4 on page 159 of [4].

Beauville's example. Let $C_{1}=C_{2}$ be the Fermat curve $x_{0}^{5}+x_{1}^{5}+x_{2}^{5}=0$ and $G$ the group $(\mathbb{Z} / 5 \mathbb{Z})^{2}$ acting on $C_{1} \times C_{2}$ by the rule

$$
\begin{aligned}
(a, b) & \cdot\left(\left[x_{0}: x_{1}: x_{2}\right],\left[y_{0}: y_{1}: y_{2}\right]\right) \\
& =\left(\left[\xi^{a} x_{0}: \xi^{b} x_{1}: x_{2}\right],\left[\xi^{a+3 b} y_{0}: \xi^{2 a+4 b} y_{1}: y_{2}\right]\right)
\end{aligned}
$$

where $\xi=e^{\frac{2 \pi i}{5}}$. Then $S=C_{1} \times C_{2} / G$ is a Beauville surface with $g_{1}=g_{2}=6$, $G^{o}=G$ and $C_{i} / G$ of type $(5,5,5)$.

In fact, Bauer and Catanese [1] have shown that when one considers all possible actions of $G$ on $C_{1} \times C_{2}$ one gets exactly two isomorphic classes of Beauville surfaces.

The relevance of Beauville surfaces lies mainly on the fact that they are the rigid ones among surfaces isogenous to a product. We recall that an algebraic variety is called rigid when it does not admit any non trivial deformation.

Most, if not all, of what is known about them is due to work done by Catanese on his own ([5]) or jointly with Bauer and Grunewald ([3], [2]).

As noted in [3] there are many interesting open problems regarding Beauville surfaces. The most obvious ones are questions such as which finite groups can occur, which curves $C_{i}$, which genera $g_{i}$, which curves or genera for a given group $G$, etc.

The following (necessarily) partial answers are known.

Theorem ([3]). 1) A finite abelian group $G$ admits an unmixed Beauville structure iff $G=(\mathbb{Z} / n \mathbb{Z})^{2},(n, 6)=1$.

2) The following groups admit unmixed Beauville structures: 
a) $A_{n}$ for large $n$.

b) $S_{n}$ for $n \in \mathbb{N}$ with $n \geq 7$.

c) $\operatorname{SL}\left(2, \mathbb{F}_{p}\right)$, $\operatorname{PSL}\left(2, \mathbb{F}_{p}\right)$ for $p \neq 2,3,5$.

In this paper we prove the following results

1) If $S=C_{1} \times C_{2} / G$ is a Beauville surface, then neither $C_{1}$ nor $C_{2}$ can be a hyperelliptic curve (Theorem 3 ).

2) The minimum genera (in the lexicographic order) that can occur in the construction of a Beauville surface is $\left(g_{1}, g_{2}\right)=(6,6)$; in other words, although in the definition of Beauville surface it is only required $g_{i} \geq 2$, one, in fact, has $g_{i} \geq 6$. The minimum being achieved by Beauville's seminal example described above (Theorem 9).

3) $S_{5}$ and $S_{6}$, hence $S_{n}$ for all $n \geq 5$, admit a Beauville structure (Corollary 15).

4) We determine which pair of genera $g_{1}, g_{2}$ occur when $G$ is $S_{5}, \operatorname{PSL}_{2}\left(\mathbb{F}_{7}\right)$ or an abelian group (Theorem 12, Theorem 13, Theorem 16).

5) Finally, we produce examples of Beauville surfaces in which $G$ is a $p$-group with $p=2,3$. These appear to be the first known examples of 2 and 3-groups admitting Beauville structure (see [2], p. 38).

\section{A criterion for $G$ to admit Beauville structures}

As shown in [2] and [3] there is a purely algebraic criterion to detect when a finite group $G$ admits a Beauville structure.

Definition 1. Let $G$ be a finite group and $a, b, c$ three generators of order $p, q, r$ respectively. We shall say that $(a, b, c)$ is a hyperbolic triple of generators if the following conditions hold:

(1) $a b c=1$,

(2) $\frac{1}{p}+\frac{1}{q}+\frac{1}{r}<1$.

Now, set

$$
\Sigma(a, b):=\bigcup_{g \in G} \bigcup_{i=1}^{\infty}\left\{g a^{i} g^{-1}, g b^{i} g^{-1}, g c^{i} g^{-1}\right\}
$$

Criterion ([2], [3]). $G$ admits an unmixed Beauville structure if and only if it has two hyperbolic triples of generators $\left(a_{i}, b_{i}, c_{i}\right)$ of order $\left(p_{i}, q_{i}, r_{i}\right), i=1,2$, satisfying the following compatibility condition:

$$
\Sigma\left(a_{1}, b_{1}\right) \cap \Sigma\left(a_{2}, b_{2}\right)=1 .
$$


The curves $C_{i}$ on which $G$ acts to produce the required Beauville surface $S=$ $C_{1} \times C_{2} / G$ arise as follows:

The triangle group

$$
\Gamma_{\left(p_{i}, q_{i}, r_{i}\right)}=\left\langle x, y, z: x^{p_{i}}=y^{q_{i}}=z^{r_{i}}=x y z=1\right\rangle
$$

acts on the upper half-plane $\mathbb{H}$ as a discrete group of isometries (i.e. as a Fuchsian group $)$ and $C_{i}=\mathbb{H} / K_{i}$ where $K_{i}$ is the kernel of the epimorphism $\theta_{i}: \Gamma_{\left(p_{i}, q_{i}, r_{i}\right)} \rightarrow$ $G$ which sends $x \rightarrow a_{i}, y \rightarrow b_{i}$ and $z \rightarrow c_{i}$. It is well known that $K_{i}$ is a torsion free group that acts freely on $\mathbb{H}$ and that, according to the Riemann-Hurwitz formula, the genus $g_{i}$ of the curve $C_{i}$ is given by

$$
2 g_{i}-2=|G|\left(1-\left(\frac{1}{p_{i}}+\frac{1}{q_{i}}+\frac{1}{r_{i}}\right)\right) .
$$

We see that $g_{i} \geq 2$ precisely because $\left(p_{i}, q_{i}, r_{i}\right)$ is of hyperbolic type.

This is a useful criterion. It permits to check through a computer program whether or not a group (of not very large order) admits Beauville structures. For instance, one has

Proposition 2. The only non abelian group of order $\leq 120$ which admits a Beauville structure is $S_{5}$.

This result has been obtained in [2] with the help of MAGMA. Incidentally, we note that $S_{5}$ is missing in the printed version of the article.

\section{Some restrictions on the product $C_{1} \times C_{2}$}

Theorem 3. Let $S=C_{1} \times C_{2} / G$ be a Beauville surface, then neither $C_{1}$ nor $C_{2}$ is a hyperelliptic curve.

Proof. Let us suppose that one of them, let us say $C_{1}$, is a hyperelliptic curve. Then, the group generated by any automorphism $\tau$ of order bigger than 2 must fix points on $C_{1}$ because otherwise the obvious quotient map $C_{1} \rightarrow C_{1} /\langle\tau\rangle$ would provide an unramified cyclic covering of degree bigger than 2 between two hyperelliptic curves. According to Maclachlan [12] this is impossible. Therefore, as $G$ acts on $C_{1} \times C_{2}$ fixed point freely, only the elements of order 2 can fix points on $C_{2}$. Hence $C_{2} / G$ would have to have type $(2,2,2)$ which is not a hyperbolic type.

Corollary 4. If $S=C_{1} \times C_{2} / G$ is a Beauville surface neither $C_{1}$ nor $C_{2}$ has genus 2 .

Proposition 5. If $S=C_{1} \times C_{2} / G$ is a Beauville surface neither $C_{1}$ nor $C_{2}$ has genus 3. 
Proof. Assume that the genus of $C_{1}$ is $g_{1}=3$. The list of triangle curves of genus 3 and their corresponding groups is well known (see e.g. [10] or [15]). In view of Proposition 2, the only group of this list which could admit a Beauville structure is $\mathrm{PSL}_{2}\left(\mathbb{F}_{7}\right)$, the simple group with 168 elements.

Now, the Beauville structures of this group are analyzed in Theorem 13 below. It is there found that the genera $g_{i}$ of $C_{i}$ have to satisfy $g_{i} \geq 8$.

Proposition 6. If $S=C_{1} \times C_{2} / G$ is a Beauville surface neither $C_{1}$ nor $C_{2}$ has genus 4.

Proof. Assume $C_{1}$ has genus 4. The list of triangle curves of genus 4 and their corresponding groups is also well known ([11], [15], see also [14]). Matching this list with Proposition 2, as we did before, we are left again with only one possibility, namely $G=S_{5}$. But $S_{5}$ can only produce a Beauville surface if the genera of $C_{1}$ and $C_{2}$ are 19 and 21 (see Theorem 12 below).

The proof of the fact that none of the curves $C_{1}$ and $C_{2}$ can have genus 5 is based on the following group theoretical result.

Proposition 7. Let $p$ be an odd prime and $G$ a group of order $2^{k} p$. Assume that $G$ maps onto a cyclic group of order $p$ or onto a dihedral group of order $2 p$. Then $G$ does not admit a Beauville structure.

Proof. Note that if $x, y$ are generators of a dihedral group of order $2 p$, then $x, y$ or $x y$ are of order $p$. Hence if $G$ is generated by $a$ and $b$, then the order of $a, b$ or $a b$ is divisible by $p$ and so a Sylow $p$-subgroup of $G$ is contained in $\langle a\rangle \cup\langle b\rangle \cup\langle a b\rangle$. Since two Sylow $p$-subgroups of $G$ are conjugated, $G$ can not admit a Beauville structure.

Corollary 8. If $S=C_{1} \times C_{2} / G$ is a Beauville surface neither $C_{1}$ nor $C_{2}$ has genus 5 .

Proof. Kuribayashi and Kimura [9] have also obtained the list of all possible groups than can occur as groups of automorphisms of a genus 5 Riemann surface. Comparing again this list with Proposition 2 we are left with only two groups, a group $G_{1}$ of order $160=2^{5} \cdot 5$ and a group $G_{2}$ of order $192=2^{6} \cdot 3$. From the description of these two groups given in [9] it is obvious that the first group maps onto a dihedral group of order 10 and the second one onto a dihedral group of order 6 . Now the result follows from Proposition 7.

Assembling together the results in this section, we obtain the following

Theorem 9. Let $C_{1}, C_{2}$ be curves of genera $g_{1}, g_{2}$ and $G$ a finite group acting on $C_{1} \times C_{2}$ so that $S=C_{1} \times C_{2} / G$ is a Beauville surface. Then $g_{1}, g_{2} \geq 6$. Furthermore, the minimum $\left(g_{1}, g_{2}\right)=(6,6)$ is achieved by Beauville's own example. 
We record in passing a collateral implication of Proposition 7

Corollary 10. Let $p$ be an odd prime and $G$ a group of order $2^{k} p$. If $(p-1) / 2$ is odd, then $G$ does not admit a Beauville structure.

Proof. Let $N=\mathrm{O}_{2}(G)$ be the maximal normal 2-subgroup of $G$. Since $G / N$ is soluble, its order is equal to $2^{l} p$ and $O_{2}(G / N)=1, G / N$ has only one minimal normal subgroup and this subgroup is of order $p$. Now, using that $(p-1) / 2$ is odd, we obtain that $G / N$ is a cyclic group of order $p$ or a dihedral group of order $2 p$. Thus, we may apply Proposition 7.

\section{The Beauville genus spectrum of a group}

Definition 11. Let $G$ be a finite group. By the Beauville genus spectrum of $G$ we mean the set $\operatorname{Spec}(G)$ of pairs of integers $\left(g_{1}, g_{2}\right)$ such that $g_{1} \leq g_{2}$ and there are curves $C_{1}, C_{2}$ of genera $g_{1}$ and $g_{2}$ with an action of $G$ on $C_{1} \times C_{2}$ such that $S=C_{1} \times C_{2} / G$ is a Beauville surface.

We observe that $\operatorname{Spec}(G)$ is always a finite set, for by the Riemann-Hurwitz formula $g_{i}$ is bounded by $1+\frac{|G|}{2}$. In fact for many groups $\operatorname{Spec}(G)=\varnothing$. For instance, the only abelian groups $G$ for which $\operatorname{Spec}(G) \neq \emptyset$ are $G=(\mathbb{Z} / n \mathbb{Z})^{2}$ with $(n, 6)=1([3])$.

We also observe that knowledge of $\operatorname{Spec}(G)$ provides knowledge of the possible numerical invariants of the Beauville surfaces $S=C_{1} \times C_{2} / G$. Indeed, as the irregularity of a Beauville surface is always $q=0$ the geometric genus $p_{g}$ is obtainable from the identity $1+p_{g}(X)-q(X)=\frac{1}{4} \chi_{\text {top }}(S)=\frac{\left(g_{1}-1\right)\left(g_{2}-1\right)}{|G|}$ (see [5]).

In this section we compute the spectrum of a few groups.

Theorem 12. $\operatorname{Spec}\left(S_{5}\right)=\{(19,21)\}$.

Proof. A Beauville surface $S=C_{1} \times C_{2} / S_{5}$ with curves $C_{1}$ of genus $g_{1}=19$ and $C_{2}$ of genus $g_{2}=21$ is provided by the following two triples of generators of $S_{5}$ :

$$
a_{1}=(125), \quad b_{1}=(13)(254), \quad c_{1}=(123)(45)
$$

and

$$
a_{2}=(2345), \quad b_{2}=(1234), \quad c_{2}=(14253) \text {. }
$$

Let now $\left(a_{i}, b_{i}, c_{i}\right)(i=1,2)$ be two compatible triples of generators of hyperbolic type $\left(p_{i}, q_{i}, r_{i}\right)$. We have to show that $\left(p_{1}, q_{1}, r_{1}\right)=(3,6,6)$ and $\left(p_{2}, q_{2}, r_{2}\right)=$ $(4,4,5)$. 
We first note that the identity $a_{i} b_{i} c_{i}=1$ implies that each of the triples $\left(a_{i}, b_{i}, c_{i}\right)$ consists of one even permutation and two odd permutations. With this observation at our disposal the rest of the proof consists of a case by case analysis of all possible types $\left(p_{i}, q_{i}, r_{i}\right)$. To simplify the task we assume that $p_{i} \leq q_{i} \leq r_{i}$. This can be done because if instead of $a_{i}, b_{i}, c_{i}$ we choose e.g. the generators $a_{i} b_{i} a_{i}^{-1}, a_{i}, c_{i}$ then the type becomes $\left(q_{i}, p_{i}, r_{i}\right)$.

We shall only consider the types with $p_{1}=2$, the remaining cases being similar.

Since the types of the form $(2,2, r),(2,3, r)$ and $(2,4,4)$ are not hyperbolic types we are left with only four possible types:

- $(2,4,5)$. Clearly $a_{1}$ has to be a transposition and $b_{1}^{2}$ a product of two disjoint transpositions. On the other hand, if $c_{2}$ has order 6 , then $c_{2}^{3}$ is conjugate to $a_{1}$. Thus, a compatible triple $\left(a_{2}, b_{2}, c_{2}\right)$ could only have type $(3,3,3)$ which is not a hyperbolic type.

- $(2,4,6)$. In this case $b_{1}^{2}$ (resp. $\left.c_{1}^{3}\right)$ would be an even (resp. odd) order two permutation. Thus, the only candidate left for the type of $\left(a_{2}, b_{2}, c_{2}\right)$ is $(5,5,5)$ which is ruled out by our observation above.

- $(2,5,6)$. In this case $a_{1}$ would have to be odd, hence the only possible type left for $\left(a_{2}, b_{2}, c_{2}\right)$ would be $(4,4,4)$ which also contradicts the main observation above.

- $(2,6,6)$. In this case $a_{1}$ would have to be even. Now, since $b_{1}^{2}$ is a permutation of order 3 and $b_{1}^{3}$ is conjugate to a transposition, the only candidate for $\left(p_{2}, q_{2}, r_{2}\right)$ is $(5,5,5)$ which, again by the observation above, is forbidden.

Theorem 13. $\operatorname{Spec}\left(\mathrm{PSL}_{2}\left(\mathbb{F}_{7}\right)\right)=\{(8,49),(15,49),(17,22),(22,33),(22,49)\}$.

Proof. The pairs of genera above correspond to the following pairs of hyperbolic type:

(i) $(3,3,4),(7,7,7)$,

(ii) $(3,4,4),(7,7,7)$,

(iii) $(3,3,7),(4,4,4)$,

(iv) $(4,4,4),(3,7,7)$,

(v) $(4,4,4),(7,7,7)$.

Obviously, any pair of triples of generators $\left(a_{i}, b_{i}, c_{i}\right)$ realizing any of these five pairs of types will be necessarily compatible. Thus, we have to show that such generating triples exist and that no other pair of hyperbolic types can be realized by a pair of compatible generating triples.

Consider the following elements of $\mathrm{PSL}_{2}\left(\mathbb{F}_{7}\right)$ :

$$
\alpha=\left(\begin{array}{cc}
-1 & -1 \\
1 & 0
\end{array}\right), \quad \beta=\left(\begin{array}{ll}
1 & 1 \\
0 & 1
\end{array}\right), \quad \gamma=\left(\begin{array}{cc}
1 & -3 \\
-3 & 3
\end{array}\right), \quad \delta=\left(\begin{array}{cc}
2 & 0 \\
1 & -3
\end{array}\right) .
$$

Next we exhibit a generating triple $\left(a, b, c=(a b)^{-1}\right)$ for each of the six types involved. 
- $(3,3,7): a=\alpha, \quad b=\beta \alpha \beta^{-1}$,

- $(7,7,7): a=\beta^{4}, \quad b=\alpha \beta \alpha^{-1}$,

- $(3,3,4): a=\beta \alpha^{2} \beta^{-1}, \quad b=\alpha$,

- $(4,4,4): a=\gamma, \quad b=\beta^{-3} \alpha$,

- $(3,7,7): a=\alpha, \quad b=\alpha \beta^{-1} \alpha^{-1}$,

- $(3,4,4): a=\alpha^{2} \delta \alpha^{-2}, b=\alpha^{2} \delta$.

Now, if one bears in mind that in $\operatorname{PSL}_{2}\left(\mathbb{F}_{7}\right)$ there is exactly one conjugacy class of elements of order 1, 2, 3, 4 and two of order 7 ([8], p. 289), it is easy to show that no other pair of types $\left(p_{i}, q_{i}, r_{i}\right)$ is possible.

While the spectrum of $S_{6}$ can be found by computer means (in fact, $\operatorname{Spec}\left(S_{6}\right)=$ $\{(49,91),(91,121),(91,169),(121,169),(151,169)\})$, a theoretical explanation of this fact similar to the one given for the groups $S_{5}$ and $\operatorname{PSL}_{2}\left(\mathbb{F}_{7}\right)$ will take too long to be included here and so we content ourselves with showing that $\operatorname{Spec}\left(S_{6}\right) \neq \varnothing$ since this is all we need to state Corollary 13.

Proposition 14. $\operatorname{Spec}\left(S_{6}\right) \neq \varnothing$

Proof. The following triples of generators produce a Beauville structure with group $S_{6}$ :

$$
\begin{array}{lll}
a_{1}=(12), & b_{1}=(123456), & c_{1}=(26543) \\
a_{2}=(1526), & b_{2}=(1234), & c_{2}=(16)(2543) .
\end{array}
$$

Corollary 15. $\operatorname{Spec}\left(S_{n}\right)$ admits a Beauville structure if and only if $n \geq 5$

By part 1 of the theorem in [3] quoted in the introduction we know that $\operatorname{Spec}(G)=$ $\emptyset$ for all abelian groups except for the groups $(\mathbb{Z} / n \mathbb{Z})^{2}$, with $(n, 6)=1$. For these exceptional groups we have

Theorem 16. For $(n, 6)=1$,

$$
\operatorname{Spec}\left((\mathbb{Z} / n \mathbb{Z})^{2}\right)=\left\{\left(\frac{(n-1)(n-2)}{2}, \frac{(n-1)(n-2)}{2}\right)\right\} .
$$

Moreover, the corresponding curves $C_{1}, C_{2}$ are both isomorphic to the Fermat curve $x_{0}^{n}+x_{1}^{n}+x_{2}^{n}=0$.

Proof. Let us denote by $F_{n}$ the Fermat curve of equation $x_{0}^{n}+x_{1}^{n}+x_{2}^{n}=0$ and by $H_{n}$ the subgroup of $\operatorname{Aut}\left(F_{n}\right)$ generated by the automorphisms

$$
\tau_{1}\left(\left[x_{0}: x_{1}: x_{2}\right]\right)=\left[\xi_{n} x_{0}: x_{1}: x_{2}\right], \quad \tau_{2}\left(\left[x_{0}: x_{1}: x_{2}\right]=\left[x_{0}: \xi_{n} x_{1}: x_{2}\right]\right.
$$

where $\xi_{n}=e^{\frac{2 \pi i}{n}}$. 
It is clear that $H_{n} \cong(\mathbb{Z} / n \mathbb{Z})^{2}$, and it is easy to see that $F_{n} / H_{n}$ is an orbifold of type $(n, n, n)$ [7].

Obviously, the proof will be a consequence of the following two statements.

1) If $(\mathbb{Z} / n \mathbb{Z})^{2}$ is generated by two elements $a, b \in(\mathbb{Z} / n \mathbb{Z})^{2}$, then $\operatorname{order}(a)=$ $\operatorname{order}(b)=\operatorname{order}(a b)=n$.

2) For any curve $C$ which, like $F_{n}$, admits a group of automorphisms $H \cong$ $(\mathbb{Z} / n \mathbb{Z})^{2}$ such that $C / H$ is an orbifold of hyperbolic type $(n, n, n)$ there is an isomorphism $\alpha: C \rightarrow F_{n}$ such that $\alpha H \alpha^{-1}=H_{n}$.

1) To prove the first statement set $\operatorname{order}(a)=k$, $\operatorname{order}(b)=m$ and consider the homomorphism

$$
\begin{aligned}
\varphi: \mathbb{Z} /(k) \times \mathbb{Z} /(m) & \rightarrow(\mathbb{Z} / n \mathbb{Z})^{2}, \\
(1,0) & \mapsto a, \\
(0,1) & \mapsto b .
\end{aligned}
$$

As $\varphi$ is an epimorphism we have $k m \geq n^{2}$, and since, by definition, $k$ and $m$ are divisors of $n$ we infer that $k=m=n$ and that $\varphi$ is in fact an isomorphism.

2) For the proof of the second statement we translate matters into the language of section 2 and so we write $C=\mathbb{H} / K, C / H=\mathbb{H} / \Gamma_{n}$ and $H=\Gamma_{n} / K$, where $\Gamma_{n}=\Gamma_{(n, n, n)}$ and $K$ is a torsion free subgroup $K \triangleleft \Gamma_{n}$. Moreover, since $H$ is abelian, we must also have $\left[\Gamma_{n}, \Gamma_{n}\right] \triangleleft K$. We then have obvious epimorphisms

$$
\Gamma_{n} /\left[\Gamma_{n}, \Gamma_{n}\right] \rightarrow \Gamma_{n} / K=H
$$

and

$$
\begin{aligned}
H \cong(\mathbb{Z} / n \mathbb{Z})^{2} & \rightarrow \Gamma_{n} /\left[\Gamma_{n}, \Gamma_{n}\right], \\
(1,0) & \mapsto \bar{\alpha}, \\
(0,1) & \mapsto \bar{\beta}
\end{aligned}
$$

where $\alpha, \beta \in \Gamma_{n}$ is any pair of elements of order $n$ generating $\Gamma_{n}$.

It follows that

$$
\left|\Gamma_{n} /\left[\Gamma_{n}, \Gamma_{n}\right]\right| \geq\left|\Gamma_{n} / K\right| \geq\left|\Gamma_{n} /\left[\Gamma_{n}, \Gamma_{n}\right]\right| .
$$

Therefore $C \cong \mathbb{H} /\left[\Gamma_{n}, \Gamma_{n}\right]$ and $H=\Gamma_{n} /\left[\Gamma_{n}, \Gamma_{n}\right]$. The conclusion is that a pair such as $(C, H)$ is unique, hence isomorphic to the Fermat pair $\left(F_{n}, H_{n}\right)$.

\section{Beauville structures on finite $p$-groups}

While for $p \neq 2,3$ the groups $(\mathbb{Z} / p \mathbb{Z})^{2}$ provide examples of $p$-groups admitting a Beauville structure, it seems that no such examples exist in the literature when $p=2,3$. The goal of this section is to construct such groups. We want to stress that 
it is very plausible that most 2 -generated finite $p$-groups of sufficiently large order have this property. Our examples have orders $2^{12}$ and $3^{12}$. It is very likely that they are not of the smallest possible order.

We will use the following notation. If $G$ is a group and $g, h$ are two elements of $G$ we denote by $g^{h}$ the element $h^{-1} g h$ and by $[g, h]$ the commutator $g^{-1} h^{-1} g h$. If $H_{1}$ and $H_{2}$ are two subgroups of $G$, then $\left[H_{1}, H_{2}\right]$ will denote the subgroup of $G$, generated by $\left\{[g, h] \mid g \in H_{1}, h \in H_{2}\right\}$. We will write $[a, b, c]$ for $[[a, b], c]$. If $n$ is a natural number, we denote by $G^{n}$ the subgroup of $G$ generated by all the $n$th powers of the elements of $G$. We also set $\Phi_{n}(G)=G^{n}[G, G]$.

Our examples will be presented as quotients of a free group. We will use the following well-known proposition.

Proposition 17. Let $F$ be a free group of rank $d, p$ a prime and $H$ a subgroup of index $n$. Then $\left|H: H^{p}[H, H]\right|=p^{(d-1) n+1}$.

Proof. This follows from the Nielsen-Schreier formula for the minimal number of generators of $H$ and the fact that $H$ is a free group.

Most of our calculations will be based on the following well-known proposition.

Proposition 18. Let $F$ be a free group with free generators $x, y_{1}, \ldots, y_{s}$. Let $N$ be a normal subgroup of $F$ such that all the $y_{i}$ lie in $N$ and $F / N$ is a cyclic group of order $n$ generated by $x N$. Then

$$
x^{n}, y_{1}, y_{1}^{x}, \ldots, y_{1}^{x^{n-1}}, \ldots, y_{s}^{x}, \ldots, y_{s}^{x^{n-1}}
$$

are free generators of $N$.

Proof. This set of generators is easily obtained by applying the Reidemeister-Schreier rewriting process (see [13] for full details)

In the following lemma we will show how this proposition may be applied.

Lemma 19. Let $F$ be a free group on free generators $x$ and $y$. Then

(i) $\left[x, y^{2}\right] \notin\left(F^{2}\right)^{2}$,

(ii) $[y, x, y, y]\left[y, x^{2}, y, y\right] \notin \Phi_{3}\left(\Phi_{3}(F)\right)$.

Proof. (i) By Proposition 18, $y^{2}, x, x^{y}$ are free generators of $\left\langle x, F^{2}\right\rangle$. Applying again Proposition 18, we see that

$$
x^{2}, y^{2},\left(y^{2}\right)^{x}, \ldots
$$

are free generators of $F^{2}$. Hence, $\left[x, y^{2}\right]=\left(\left(y^{2}\right)^{x}\right)^{-1} y^{2} \notin\left(F^{2}\right)^{2}$. 
(ii) Using Proposition 18 we obtain that $x^{3}, y, y^{x}, y^{x^{2}}$ are free generators of $\left\langle y, \Phi_{3}(F)\right\rangle$. Hence, $x^{3}, y,[y, x],\left[y, x^{2}\right]$ are also free generators of $\left\langle y, \Phi_{3}(F)\right\rangle$. Applying again Proposition 18 we see that

$$
y^{3},[y, x],[y, x]^{y},[y, x]^{y^{2}},\left[y, x^{2}\right],\left[y, x^{2}\right]^{y},\left[y, x^{2}\right]^{y^{2}}, x^{3}, \ldots
$$

are free generators of $\Phi_{3}(F)$. Note that $[y, x, y, y] \in H_{1}=\left\langle[y, x],[y, x]^{y},[y, x]^{y^{2}}\right\rangle$ and $[y, x, y, y] \notin \Phi_{3}\left(\Phi_{3}(F)\right)$. Also $\left[y, x^{2}, y, y\right] \in H_{2}=\left\langle[y, x],[y, x]^{y},[y, x]^{y^{2}}\right\rangle$ and $\left[y, x^{2}, y, y\right] \notin \Phi_{3}\left(\Phi_{3}(F)\right)$. Thus, $[y, x, y, y]\left[y, x^{2}, y, y\right] \notin \Phi_{3}\left(\Phi_{3}(F)\right)$.

Theorem 20. There is a 2-group $G$ of order $2^{12}$ which admits a Beauville structure.

Proof. Let $F$ be a free group of rank 2 generated by $x$ and $y$. Denote by $H$ the normal subgroup generated (as a normal subgroup of $F$ ) by $\left\{x^{2}, y^{2},(x y)^{4}\right\}$. Then $H$ has index 8 in $F$ (note that $F / H$ is isomorphic to the dihedral group of order 8). Put $N=H^{2}=H^{2}[H, H]$ and $G=F / N$. Then, by Proposition 17, $G$ has order $2^{12}$.

Put $a=x N, b=y N, a_{1}=a b^{2}$ and $b_{1}=b(a b)^{4}$. We want to show that $\Sigma(a, b)$ and $\Sigma\left(a_{1}, b_{1}\right)$ have a trivial intersection. Since the order of all elements from $\Sigma(a, b)$ and $\Sigma\left(a_{1}, b_{1}\right)$ is a power of 2 and these two sets are closed by taking powers, it is enough to compare only elements of order 2 . The elements of order 2 in $\Sigma(a, b)$ are the conjugacy classes of $a^{2}, b^{2},(a b)^{4}$ and the elements of order 2 in $\Sigma\left(a_{1}, b_{1}\right)$ are the conjugacy classes of $a_{1}^{2}, b_{1}^{2},\left(a_{1} b_{1}\right)^{4}$.

Let $K=H / N$ and $L=[K, G]$. Note that $a^{2} L$ is not conjugate to $b_{1}^{2} L=b^{2} L$ and $\left(a_{1} b_{1}\right)^{4} L=(a b)^{4} L$ in $G / L$, because these three elements are central in $G / L$ and they are different. Using the same argument, we conclude that $b^{2}$ is not conjugate to $a_{1}^{2}$ and $\left(a_{1} b_{1}\right)^{4}$ and $(a b)^{4}$ is not conjugate to $a_{1}^{2}$ and $b_{1}^{2}$.

Thus, we only have to check that $a^{2}$ is not conjugate to $a_{1}^{2}, b^{2}$ is not conjugate to $b_{1}^{2}$ and $(a b)^{4}$ is not conjugate to $\left(a_{1} b_{1}\right)^{4}$.

Since the centralizer $C_{G}\left(a^{2}\right)$ of $a^{2}$ in $G$ is $\langle a, K\rangle$, the conjugacy class of $a^{2}$ consists of four elements:

$$
\left(a^{2}\right)^{G}=\left\{a^{2},\left(a^{2}\right)^{b},\left(a^{2}\right)^{[b, a]},\left(a^{2}\right)^{b a}\right\} .
$$

Note that $C_{G}\left(a_{1}^{2}\right)=\left\langle a_{1}, K\right\rangle=C_{G}\left(a^{2}\right)=C_{G}\left(a^{2}\right)^{[a, b]}, C_{G}\left(a_{1}^{2}\right) \neq C_{G}\left(a^{2}\right)^{b}$ and $C_{G}\left(a_{1}^{2}\right) \neq C_{G}\left(a^{2}\right)^{b a}$. Hence, $a_{1}^{2} \neq\left(a^{2}\right)^{b}$ and $a_{1}^{2} \neq\left(a^{2}\right)^{b a}$.

Observe, now, that $a^{2} \equiv\left(a^{2}\right)^{[b, a]}\left(\bmod \left(G^{2}\right)^{2}\right)$ and from part (i) of Lemma 19 it follows that $a_{1}^{2}=\left(a b^{2}\right)^{2}=a^{2}\left[a, b^{2}\right] \not \equiv a^{2}\left(\bmod \left(G^{2}\right)^{2}\right)$. Thus, $a_{1}^{2} \notin\left(a^{2}\right)^{G}$.

We have that $\left(b_{1}\right)^{2}=\left(b(a b)^{4}\right)^{2}=b^{2}\left[b,(a b)^{4}\right]$. Note that $\left[b,(a b)^{4}\right]$ commutes with $a b$ and is different from 1 . On the other hand, the conjugacy class of $b^{2}$ consists of four elements:

$$
\left(b^{2}\right)^{G}=\left\{b^{2}\left[b^{2},(a b)^{i}\right] \mid i=0,1,2,3\right\}
$$

and $\left[b^{2},(a b)^{i}\right]$ commutes with $a b$ if and only if $i \equiv 0(\bmod 4)$. Thus, $b_{1}^{2} \notin\left(b^{2}\right)^{G}$. 
Finally, we have that

$$
\left((a b)^{4}\right)^{G}=\left\{(a b)^{4},\left((a b)^{4}\right)^{a}=(b a)^{4}\right\}
$$

and

$$
\begin{aligned}
\left(a_{1} b_{1}\right)^{4} & =\left(a b^{2} b(a b)^{4}\right)^{4}=\left((a b)^{5} b^{2}\right)^{4} \\
& =(a b)^{4}\left[b^{2},(a b)^{5},(a b)^{5},(a b)^{5}\right]=\left(a b^{-1}\right)^{4}
\end{aligned}
$$

Note that $x y, y x, x y^{-1}$ form a free set of generators of $\left\langle x y, F^{2}\right\rangle$ and so

$$
(x y)^{4},(y x)^{4},\left(x y^{-1}\right)^{4}
$$

are a part of a free set of generators of $H$. Thus, $(a b)^{4},(b a)^{4},\left(a b^{-1}\right)^{4}$ are different, whence $\left(a_{1} b_{1}\right)^{4} \notin\left((a b)^{4}\right)^{G}$.

Theorem 21. There is a 3-group $G$ of order $3^{12}$ which admits a Beauville structure.

Proof. Let $F$ be a free group of rank 2 generated by $x$ and $y$ and $N=\Phi_{3}\left(\Phi_{3}(F)\right)$. We put $G=F / N$. Then, by Proposition $17, G$ has order $3^{12}$.

Put $a=x N, b=y N, a_{1}=a b^{3}$ and $b_{1}=b[a, b]$. We want to show that $\Sigma(a, b)$ and $\Sigma\left(a_{1}, b_{1}\right)$ have a trivial intersection. Arguing as in the proof of the previous theorem, we see that it is enough to show that $a^{3}$ is not conjugate to $a_{1}^{3}, b^{3}$ is not conjugate to $b_{1}^{3}$ and $(a b)^{3}$ is not conjugate to $\left(a_{1} b_{1}\right)^{3}$.

For example, let us see that $b^{3}$ is not conjugate to $b_{1}^{3}$. First note that

$$
b_{1}^{3}=(b[a, b])^{3}=b^{3}[a, b, b, b] .
$$

On the other hand

$$
\left(b^{3}\right)^{G}=\left\{b^{3},\left(b^{3}\right)^{a}=b^{3}[b, a, b, b],\left(b^{3}\right)^{a^{2}}=\left[b, a^{2}, b, b\right]\right\} .
$$

Note that $[a, b]^{-1}=[b, a]$. Since $[a, b, b, b] \neq 1$,

$$
[a, b, b, b]=[b, a, b, b]^{-1} \neq[b, a, b, b]
$$

and so $b_{1}^{3} \neq\left(b^{3}\right)^{a}$.

We can now use part (ii) of Lemma 19 to obtain that $b_{1}^{3} \neq\left(b^{3}\right)^{a^{2}}$. Thus, we conclude that $b_{1}^{3} \notin\left(b^{3}\right)^{G}$. The other cases are proved in a similar way.

Corollary 22. For every prime number $p$ there is a p-group $G$ which admits a Beauville structure.

Acknowledgment. 1) We thank the referee for many interesting comments including several suggestions for future research. We also thank him for the computation of the full genus spectrum of the group $S_{6}$ which agrees with the result found for us by our colleague Ernesto Girondo by means of GAP.

2) The research of the three authors has been supported by the Spanish Ministry of Science and Innovation, grants MTM2006-01859, MTM2006-14688 and MTM200806680 and the Universidad Autónoma de Madrid, grant CCG08-UAM/ESP-4145. 


\section{References}

[1] I. Bauer and F. Catanese, Some new surfaces with $p_{g}=q=0$. In The Fano Conference: organized to commemorate the 50th anniversary of the death of Gino Fano (1871-1952), Università di Torino, Turin 2004, 123-142. Zbl 1078.14051 MR 2112572

[2] I. Bauer, F. Catanese, and F. Grunewald, Beauville surfaces without real structures. In Geometric methods in algebra and number theory, Progr. Math. 235, Birkhäuser, Boston 2005, 1-42. Zbl 1094.14508 MR 2159375

[3] I. Bauer, F. Catanese, and F. Grunewald, Chebycheff and Belyi polynomials, dessins d'enfants, Beauville surfaces and group theory. Mediterr. J. Math. 3 (2006), 121-146. Zbl 1167.14300 MR 2241319

[4] A. Beauville, Surfaces algébriques complexes. Astérisque 54 (1978), 1-172. Zbl 0394.14014 MR 0485887

[5] F. Catanese, Fibred surfaces, varieties isogenous to a product and related moduli spaces. Amer. J. Math. 122 (2000), 1-44. Zbl 0983.14013 MR 1737256

[6] G. González-Diez, Belyi’s theorem for complex surfaces. Amer. J. Math. 130 (2008), 59-74. Zbl 1158.14015 MR 2382142

[7] G. González-Diez, R. A. Hidalgo, and M. Leyton, Generalized Fermat curves. J. Algebra 321 (2009), 1643-1660. Zbl 1175.14023 MR 2498261

[8] I. M. Isaacs, Character theory of finite groups. Pure Appl. Math. 69, Academic Press, New York 1976. Zbl 0337.20005 MR 0460423

[9] A. Kuribayashi and H. Kimura, Automorphism groups of compact Riemann surfaces of genus five. J. Algebra 134 (1990), 80-103. Zbl 0709.30037 MR 1068416

[10] A. Kuribayashi and K. Komiya, On the structure of the automorphism group of a compact Riemann surface of genus 3. Bull. Fac. Sci. Engrg. Chuo Univ. 23 (1980), 1-34. Zbl 0464.30036 MR 626109

[11] A. Kuribayashi and I. Kuribayashi, A classification of compact Riemann surfaces of genus four. Bull. Fac. Sci. Engrg. Chuo Univ. 26 (1983), 25-42. Zbl 0574.30044 MR 742692

[12] C. Maclachlan, Smooth coverings of hyperelliptic surfaces. Quart. J. Math. Oxford Ser. (2) 22 (1971), 117-123. Zbl 0208.10101 MR 0283194

[13] W. Magnus, A. Karrass, and D. Solitar, Combinatorial group theory. Interscience Publishers, New York 1966. Zbl 0138.25604 MR 0207802

[14] G. Riera and R. E. Rodríguez, The period matrix of Bring's curve. Pacific J. Math. 154 (1992), 179-200. Zbl 0734.30036 MR 1154738

[15] J. Wolfart, Triangles groups and Jacobians of CM type. Manuscript, Frankfurt a. M. 2000. http://www.math.uni-frankfurt.de/ wolfart/Artikel/jac.pdf

Received September 25, 2008; revised January 12, 2010

Y. Fuertes, G. González-Diez, A. Jaikin-Zapirain, Departamento de Matemáticas, Universidad Autónoma de Madrid, Ciudad Universitaria de Cantoblanco, Madrid 28049, Spain E-mail: yolanda.fuertes@uam.es; gabino.gonzalez@uam.es; andrei.jaikin@uam.es 